\title{
A New Paradigm for a New Era The Cosmic Dark Matter Fractal Field Theory
}

\author{
Timothy Fulton Johns*
}

Decathlon Dental, USA

Received: 漹 July 10, 2018; Published: 阱July 24, 2018

*Corresponding author: Timothy Fulton Johns, Decathlon Dental, 614 Mabry Hood Road \#201 Knoxville, TN 37932, USA, Tel: (865)

675-3773; Email: tfjohns@gmail.com

\section{Opinion}

There is always more than meets the eye in a fractal frame of reference. Almost three quarters of our globe is home to another world we are only beginning to understand. Yet what has been very obvious from our earliest investigation of the sea is that it reveals a complex reality alien to us. The sea is a world in and of itself. Though it provides the very fabric of existence of the life it sustains, the combined network of oceans that blankets our planet goes unnoticed by its inhabitants due to its scale and the fact that these waters are the very space (i.e., fluid) these life-forms live in and navigate through moment by moment. As it is with the sea life, so it is in our universe. We, like our marine counterparts, fail to notice the sea of our own reality. The space we occupy and live in is empty to our everyday sensory perceptions until of course we feel the wind on our face. Only then do we become aware that something unseen and unrealized actually exist. The five senses we possess are incapable of detecting the extremes of both subatomic and galactic scales, yet they do exist, and that is the difference in my view between what we call our universe and the cosmos. That which we know about and can perceive or measure is our universe. That which is beyond our current knowledge and our perception is what I refer to as the cosmos.

Much of our cosmos is out of our current knowledge and limited sensory perception, but our universe is inclusive within the cosmos. Mankind has now reached out deep into the universe probing both extremes in many different ways looking for answers too many questions but especially to an age old question, Are we alone? Is it just us in this entire abyss? It is my opinion a new paradigm is needed to truly probe the largest and especially the smallest zones of our cosmos to answer such questions. This will require a new theory to compare, test and possibly rethink current accepted beliefs about our universe, as well as, answers to questions that science has yet to explain at the Planck extremes. I am purposing such a theory, which so far has provided new ways to understand our reality. I have written five papers based on this new perspective thus far, three of which have been published expounding on this theory and I will provide links for your review. The Cosmic Dark Matter Fractal Field Theory provides a workable model that reveals that many current widely held conclusions regarding many accepted observations about our universe may be wrong.

I will briefly discuss one for an example, that the universe is expanding and even accelerating; this new theory reveals that this conclusion is possibly being misinterpreted. This is in part because of two problems that seem to be insurmountable. First and foremost is that this theory is based on such a small sample size; only $4 \%$ of the known total of our universe contains ordinary matter, baryonic matter. Which is also being viewed and evaluated from only one perspective of a multi-scalar continuum. Therefore, any findings based on such a small sample size of the known total and limited scalar perspective is subject to very questionable credibility no matter what your evidence. This entire arena of inquiry is without foundation given dark matter/dark energy (DM/DE) which represents $96 \%$ of the subject under investigation and has no workable model in place to use for understanding its nature and makeup. Second, there is no map, no theory that unifies the functional and structural components to give a vision which leads to an understanding of the dynamics of the known expanse of our cosmos from the Planck quantum scale $10^{\wedge}-35$ to the Newtonian based galactic scale $10^{\wedge} 35$; that can be used as a model or framework for evaluation, comparison and analysis of observational and experimental data collected. What is interpreted as expansion is in all probably actually a flow; a literal independent motion of space-time, what I call the Great Cosmic Sea of Reality. This flow is possibly emanating from the Planck scale through all scales up to the most super-massive black holes.

There is also possibly a refractive distortion of the common probe used in cosmology, light. This probe is very ambiguous because it is subject to distortion, due to gravity. There is the potential for a large gravity shift at the interface of two very different worlds the Baryonic Matter/ Dark Matter Dark Energy interface with two extremes of gravitational influence across a barrier, a zone of demarcation between these zones distorting the path of light, as explained in The Cosmic Dark Matter Fractal Field Theory (CDMFFT) in the book called The Great Cosmic Sea of Reality. We reside in just one zone in the exact center of a large 
scalar continuum from $10^{\wedge}-35$ to $10^{\wedge} 35$ and everything we look at, probe and measure is from that perspective. Anyone of these facts is very likely to influence the results of any conclusion about expansion of our universe so I propose that widely accepted conclusion be revisited with a different model in place.
ISSN: 2574-1241

DOI: 10.26717/BJSTR.2018.07.001463

Timothy Fulton Johns. Biomed J Sci \& Tech Res

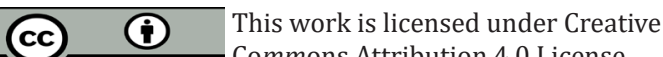

Submission Link: https://biomedres.us/submit-manuscript.php

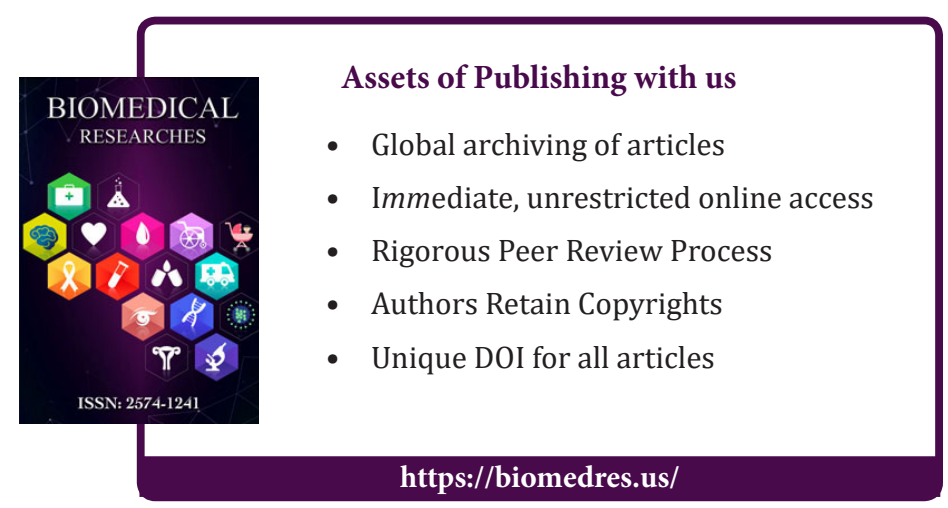

